\title{
Does physiotherapy applied in conjunction with compression brace treatment in patients with pectus carinatum have efficacy? A randomized controlled study
}

Nuray ALACA ( $\nabla$ nuray.alaca@acibadem.edu.tr)

Acibadem Mehmet Ali Aydinlar Universitesi https://orcid.org/0000-0003-3034-9388

insan ALACA

Pectus asociation

Mustafa Yüksel

pectus asocciation

Research article

Keywords: Pectus carinatum, braces, rehabilitation, exercise therapy

Posted Date: May 3rd, 2020

DOI: https://doi.org/10.21203/rs.3.rs-25151/v1

License: (c) (1) This work is licensed under a Creative Commons Attribution 4.0 International License.

Read Full License

Version of Record: A version of this preprint was published at Pediatric Surgery International on May 18th, 2020. See the published version at https://doi.org/10.1007/s00383-020-04675-3. 


\section{Abstract}

Background Non-invasive treatment of pectus carinatum (PC) deformity includes the use of a compression brace and exercises. In this study, we aimed to examine the effect of a physiotherapy protocol applied as adjunct to compression brace treatment in patients with PC.

Methods The study included 30 male patients between 11-18 years of age. Patients were randomly assigned into two groups: a brace treatment only group (Group 1) and a brace and physiotherapy group (Group 2). Patient demographics and disease related properties, protrusion measurements, postural evaluations, deformity perceptions, life quality and treatment satisfaction were evaluated (before and after 12 weeks).

Results Although both groups showed improvements based on external chest measurements related to PC protrusion following treatment $(p<0.001)$, Group 2 had more benefit from the treatment (effect size > $0.36)$ and displayed greater improvement in maximum protrusion degree and lateral length values $(p<$ 0.05). Additionally, we found that patient perception of deformity, posture, psychological life quality, and treatment satisfaction scores were significantly better in Group $2(p<0.05)$.

Conclusion Owing to the satisfaction and additional benefits observed in the physiotherapy group, we think a proper cardiopulmonary and musculoskeletal exercise program should be applied concurrently with brace treatment for patients with PC deformity. Nevertheless, long- term outcomes need to be clarified in future studies.

Trial registration:

The study was approved by the ethics committee (ATADEK-2019-14/11) and retrospectively registered as a clinical trial (ClinicalTrials.gov Identifier: NCT04167800, 20 November 2019)

\section{Background}

The development of the rib, cartilage, and sternum that form the chest wall with various abnormalities of the musculoskeletal system, or isolated, is called chest wall deformities [1]. Among these, protrusion of the anterior chest wall caused by sternum and costal cartilages is called pectus carinatum (PC, pigeon chest) and comprises the second most common type of chest wall deformity [2]. Slightly noticeable at birth, it often becomes apparent later (11-15 years of age). In adolescence, the deformity becomes prominent with an accelerated growth rate. Most patients are asymptomatic. Respiratory function tests do not indicate significant pathology. In case of accompanying scoliosis, restrictive respiratory function disturbance can be detected $[1,3]$. Rather than physical symptoms, the deformity causes cosmetic and psychosocial problems. Individuals with PC have to deal with self-consciousness, embarrassment, anxiety, depression, and social isolation. Patients may have negative body esteem and poor life quality [4]. Additionally, patients have postural distortion, which can be aggravated by leaning forward, a habitual behavior patients often do while trying to hide their deformity [5]. 
Until recently, surgery has been the basis of treatment in PC. The most widely recognized and applied techniques are the Ravitch procedure and modifications include the subperichondreal resection of cartilage as well as sternal reconstruction [6]. Currently, this technique can also be performed thoracoscopically [7]. Nonetheless, an alternative and new surgical technique, the Abramson procedure (also called inverted Nuss procedure) has recently gained significance in recent years owing to its less invasive nature [8]. It involves subcutaneous placement of a steel rod over the sternum. Mounting of this rod to ribs helps to restore normal thoracic shape. Nevertheless, surgical corrections also come with some disadvantages including the need for hospitalization as well as the risk of adverse effects such as wound scars, pneumothorax, wound infection, recurrence, skin necrosis and high treatment costs [9]. These disadvantages have caused many patients and families to face a dilemma between undergoing a major operation or not being treated at all; thus, surgical correction has often been spared to the most severe patients. Patients with mild or moderate PC were usually not corrected with surgery [10]. The ability to correct the deformity with surgical techniques showed plasticity of the chest wall, and led to the presumption that the deformity could be reshaped with chronic compression. This plasticity could be the target of non-operative treatment [11].

Noninvasive treatment methods for PC include compression braces and exercise programs that target the deformity itself as well as the concurrent postural distortion and scoliosis. Compression braces can be introduced as a conservative treatment method for PC due to the fact that the chest wall remains flexible during adolescence and can be restored to normal position by applying external compression on the sternum and costal cartilage. Compression braces can be applied according to the flexibility of the deformity [11]. Flexibility can be assessed by a manual compression test or measurement of the correction pressure via a device. Patients eligible for orthotic correction have been defined as those with a chondrogladiolar type deformity who require less than 10-15 psi pressure for correction [4]. Side effects of the compression brace include erythema, itching, wound, dyspnea, back pain, chest pain, and feeling of discomfort and embarrassment [12]. Even with a lack of brace standardization, patients are recommended to wear brace for 23 hours according to Calgary protocol. After achievement of correction, the use of the brace is confined to night hours until completion of the growth of axial skeleton [11]. Most of evidence regarding this non-invasive treatment method comes from retrospective or prospective case series. In one prospective series, patients wearing braces were advised to perform chest wall musclestrengthening exercises, but the effects of the exercises on treatment results were not investigated [13]. Exercises have also been reported to contribute to the concealment of the deformity by strengthening the muscles [11]. Therefore, in this study, we aimed to investigate the effect of our physiotherapy protocol that combines cardiopulmonary and musculoskeletal physiotherapy applied in conjunction with the compression brace treatment in patients with PC.

\section{Materials And Methods}

The study was designed as a randomized and prospective study. The study was approved by the ethics committee (ATADEK-2019-14/11) and registered as a clinical trial (registration no: NCT04167800). A total of 30 patients between the ages of 11 and 18 years old who presented to the Pectus Association with 
discontent about their deformities and brace indication were invited to the study. These 30 patients had either a symmetrical or asymmetrical chondrogladiolar type of PC deformity. The inclusion criteria for the study are a deformity requiring corrective pressure of less than $10 \mathrm{psi}$ in compression test, use of the device for the first time, and/or volunteering to participate in the study. All patients were diagnosed and evaluated for brace indication by a thoracic surgeon with 30 years of experience. Study exclusion criteria were history of previous brace use, severe scoliosis (Cobb angle above 20 degrees), chronic systemic disease or serious psychiatric illness, complex mixed type pectus deformity, and unwillingness to use brace.

Sample size was determined with GPower 3.1 software (Kiel University, Kiel, Germany) to achieve a 95\% power ratio with a 0.05 margin of error within $95 \%$ confidence interval. Based on our calculations, we needed 8 patients in each group for a total of 16 patients $[14,15]$. In consideration of the possible lack of compliance with brace use or exercise, we decided to include 15 patients in each group. Patients were randomized into 2 groups with block randomization technique using Random Allocation Software.

Group 1: Patients who used a compression brace 23 hours a day for a 12 week duration (n:15) Group 2: Patients who used a compression brace 23 hours a day and attended a physiotherapy program for a 12 week duration (n:15)

After instructing all patients on how to use the appropriate compression brace, they were advised to wear it for 23 hours a day for a period of 12 weeks. Regular use was monitored by advising the patient's relative to keep a record of daily use. Those who did not wear the brace for 5 consecutive days were excluded from the study. The second group of patients received a physiotherapy protocol that was comprised of a combination of cardiopulmonary and musculoskeletal physiotherapy. Once a week, exercise treatment was applied by physiotherapists who had at least 20 years of experience in cardiopulmonary (IA) and musculoskeletal physiotherapy (NA). Patients followed an in-home exercise program for the remaining days of the week. Patients were asked to perform musculoskeletal exercises at least 3-4 times a week and respiratory exercises with 10-12 repeats $2-3$ times a day. Patients were allowed to record the instruction of exercises with a camera to help them repeat the exercises by watching them again. Patient relatives were asked to keep a record of exercises to monitor compliance. Patients who failed to perform exercise sessions for 5 consecutive days were excluded from the study. All treatments were continued for a period of 12 weeks.

\section{Rehabilitation program}

Exercise density during rehabilitation was determined according to the Borg scale [16], and intensified gradually according to the patient's condition. Pulmonary exercises formed the basis of the rehabilitation program in this study, and included the following methods ( 25 minutes for each physiotherapy session):

Diaphragmatic respiration

Lower lateral costal respiration 
Middle and upper lateral costal respiration (in case of asymmetrical PC, trying to avoid inhalation to the side of protrusion, and expansion to the other side)

Respiratory exercises separately for each lung lobe (in case of asymmetrical PC, trying to avoid inhalation to the side of protrusion, and expansion to the other side)

Respiratory exercise to the posterior lower and middle lobes in the prone position

Working a combination of above exercises while holding breath

All respiratory exercises were instructed with manual guidance from the physiotherapist. Afterwards, no manual guidance was provided to patients who learned these exercises properly.

Musculoskeletal exercises were applied following evaluation of the patients' postural distortion, muscle shortness and weakness, and were based on the program described below (twice a day and 3-4 times a week). Exercises targeting the whole musculoskeletal region were always applied according to the patient's deformity in combination with segmental respiratory exercises and based on the patient's conditions (25 minutes for each physiotherapy session).

Stretching exercises to the cervical region and lateral flexors (particularly scalene muscles, sternocleidomastoid, and upper trapezius) and latissimus dorsi muscles (initially 5-10 seconds, then 30 seconds, 3-5 repeats). Afterwards, strengthening exercises to accessory respiratory muscles with respiration.

Stretching pectoralis major muscle and then strengthening the sternocostal part of pectoralis major muscle (in case of asymmetrical PC, stretching pectoralis major muscle located at the side of protrusion, strengthening to the other side)

Strengthening exercises to back extensors, lower and middle sections of trapezius, rhomboids, serratus anterior, serratus posterior inferior and rotator cuff muscles

Strengthening rectus abdominus and lower and oblique abdominal muscles

Posterior tilting of cervical and lumbar regions

Strengthening quadratus lumborum (asymmetrical) (based on patient's condition)

To increase mobility of the spine and thorax, manipulation and mobilization methods were applied to proper regions in vertebras, and mobilization was applied to sternocostal joints and area of protrusion. Additionally, postural awareness exercises were performed along with respiratory exercises, while standing in front of a mirror (10 minutes for each physiotherapy session).

Following the first month of treatment (after respiratory exercises were learned and thoracic mobility was increased), patients were recommended to do walking or jogging for 30 minutes, 5 times a week, based on American College of Sports Medicine guidelines as to keep maximum heart rate (220-age) between $60 \%$ and $80 \%$ (moderate intensity) [17].

\section{Evaluations}

The subjects age, height, weight, deformity characteristics (mild, moderate, severe, and symmetrical/asymmetrical), complaints, and side effects of orthotics were recorded [18]. Medical history, 
family history, postural disturbances, avoidance of doing sports/swimming, fatigue, tendency to put on more clothes, and embarrassment due to chest condition were questioned and noted. The following evaluations were made before and after treatment.

\section{External chest wall measurements}

Anthropometric measurements were performed as described by Lee et al. [14] and included maximum protrusion angle, craniocaudal length, and lateral length. Maximum protrusion angle was recorded as the distance between the point of maximum protrusion to the estimated normal chest wall level. Craniocaudal length was measured as the length of the protruded area at the point of maximum protrusion. Lateral length was measured as the horizontal length of the area at the point of maximum protrusion.

Additionally, caliper measurements described by Ewert et al. [19] were performed. These included:

T1: between upper edge of manubrium - spinous process of the vertebra at the same level T2: between Ludovici angle - spinous process of the vertebra at the same level T3: between point of maximum protrusion at the sternum - spinous process of the vertebra at the same level.

PC severity was calculated with the formula: T3/T1 $\times 100(\%)$. Patients are defined as PC if the result is greater than 140. The T3 measurement and PC severity show the highest correlation with Haller and correction index [19].

\section{Patient's perception of deformity}

Patient's perception of deformity was evaluated with self-scoring of the chest image ( $0-10$ points) as described by Canavan and Cahalin [20]. A 0 point means the worst possible perception, and a 10 point means the best possible perception of chest image.

\section{Pain evaluation}

Pain experienced by the patient during use of the brace was assessed with visual analog scale (VAS). The patient was asked to mark the level of pain on a vertical scale of $10 \mathrm{~cm}$ in length. The lowermost point (0) indicated minimum pain and the uppermost point (10) indicated maximum pain. The patient's marking on the VAS scale reflected his/her pain level [21].

\section{Postural evaluation}

Patients' postures were assessed with New York Posture Rating Scale (NYPR). Using this scale, possible posture changes in 13 body alignment segments were assessed and scored. Accordingly, correct posture was scored as 5 points, slight deviation as 3 points and pronounced deviation as 1 point. The maximum available score was 65 , and the minimum was 13 . Standard evaluation criteria for this scale were defined as very good for total score $\geq 45$, good for $40-44$, fair for $30-39$, poor for $20-29$, and bad for $\leq 19$ [22]. 
Treatment satisfaction was assessed with Global Rating of Change (GRC) scale. GRC was scored on a 5 point Likert scale with the following responses: much better $(+2)$, better $(+1)$, same $(0)$, worse $(-1)$, and much worse (-2) [23].

\section{Evaluation of life quality}

Pectus Deformity Life Quality Questionnaire is used to assess disease specific life quality of patients with pectus deformity and their parents. The first validation study of the questionnaire was conducted by Lawson et al. [24]. Krasopoulos et al. [25] modified the questionnaire by reversing the scoring for the first 3 items. It was translated into Turkish by Bahadir et al [26]. The questionnaire was made up of 12 items, and the parental form was made up of 13 items. Each item was scored on a scale of 4 points, where higher points indicated better life quality. Items 1-9 in patient form and items 1-8 in parental form assess psychosocial life quality, whereas items 10-12 in patient form and items $9-13$ in parental form assess physical life quality.

\section{Statistical Analysis}

Statistical analyses were made using SPSS-21.0 software (SPSS Inc., Chicago, IL). Baseline demographic data were compared between treatment groups using independent sample t-test and chi-square test for continuous and categorical variables. Pre-treatment and post-treatment values within the groups were compared with paired sample t-test. Treatment evaluations were made in consideration of inter-subject factor and time (before and after), using $2 \times 2$ mixed model analysis of variance (ANOVA). For all tests, statistical level of significance was determined as a p-value of less than 0.05 .

For each treatment, effect size (ES) was calculated using Cohen's formula. For within group comparisons, ES values of $0.2,0.5$, and 0.8 were accepted as small, moderate, and big, respectively $[27,28]$.

\section{Results}

Three patients in Group 1 did not want to continue wearing brace due to pain and fear of noticeability; therefore, they left the study. Two patients stated that they only wanted to wear the brace when they were at home or sleeping because they could not pay attention in school due to pain and fear that the brace could be noticed; therefore, they were excluded from the study. In Group 2, one patient wanted to wear the brace only outside the school, and one patient did not fully comply with the exercises; these two patients were excluded. Thus, the study was completed with 10 patients in Group 1 and 13 patients in Group 2.

Demographic and disease-related properties did not show any statistically significant difference between the groups at the start of the study ( $p>0.05$, Table 1). Maximum protrusion degree and lateral length showed statistically significant reduction within both groups $(p<0.001$, Table 2$)$. In comparison of the two groups, maximum protrusion degree and lateral length showed a significantly greater reduction in Group $2(p<0.05)$. Craniocaudal length showed a statistically significant reduction in Group $2(p=0.039)$, whereas no significant change was observed in Group $1(p=0.453)$. Regarding caliper measurements, the 
T3 region and PC severity values showed a statistically significant reduction in both groups $(p<0.01$, Table 2). 
Table 1

Demographic and disease related properties of patients

\begin{tabular}{|c|c|c|c|}
\hline Parameters & $\begin{array}{l}\text { Group } 1 \\
\text { [mean } \pm \text { standard deviation or } n \\
\text { (frequency \%)], n:10 }\end{array}$ & $\begin{array}{l}\text { Group } 2 \\
\text { [mean } \pm \text { standard deviation or } n \\
\text { (frequency \%)], } \mathrm{n}: 13\end{array}$ & 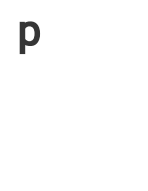 \\
\hline Age & $13.66 \pm 1.49$ & $13.90 \pm 1.44$ & 0.670 * \\
\hline Body Mass Index & $15.32 \pm 2.24$ & $15.51 \pm 2.02$ & $0.622^{\star}$ \\
\hline $\begin{array}{l}\text { Asymmetric PC } \\
\text { Deformity }\end{array}$ & $4(40 \%)$ & $7(53.84 \%)$ & 0.510 \\
\hline \multicolumn{4}{|l|}{ Deformity degree } \\
\hline Mild & $3(30 \%)$ & $5(38.46 \%)$ & \multirow[t]{3}{*}{0.902} \\
\hline Moderate & $5(50 \%)$ & $6(46.15 \%)$ & \\
\hline Severe & $2(20 \%)$ & $2(15.38 \%)$ & \\
\hline \multicolumn{4}{|l|}{ Complaint } \\
\hline Cosmetic & $8(80 \%)$ & $11(84.61 \%)$ & \multirow[t]{2}{*}{0.273} \\
\hline $\begin{array}{l}\text { Cosmetic + } \\
\text { Shortness of } \\
\text { breath }\end{array}$ & $2(20 \%)$ & $2(15.38 \%)$ & \\
\hline \multicolumn{4}{|l|}{ Clinical Symptoms } \\
\hline Tiredness & $1(10 \%)$ & $2(15.38 \%)$ & 0.385 \\
\hline Being ashamed & $3(30 \%)$ & $3(23.07 \%)$ & 0.708 \\
\hline $\begin{array}{l}\text { To dress too much } \\
\text { clothes. }\end{array}$ & $2(20 \%)$ & $3(23.07 \%)$ & 0.859 \\
\hline Avoiding sports & $2(20 \%)$ & $1(7.69 \%)$ & 0.704 \\
\hline \multicolumn{4}{|l|}{ Posture disorders } \\
\hline Forward head & $10(100 \%)$ & $13(100 \%)$ & \\
\hline Rounded shoulder & $10(100 \%)$ & $12(92.30 \%)$ & 0.370 \\
\hline $\begin{array}{l}\text { Shoulder height } \\
\text { difference }\end{array}$ & $9(90 \%)$ & $12(92.30 \%)$ & 0.846 \\
\hline Kyphosis & $8(80 \%)$ & $12(92.30 \%)$ & 0.385 \\
\hline
\end{tabular}

PC: Pectus carinatum; Group 1, patients who wore compression brace for 23 hours a day for a period of 12 weeks; Group 2, patients who wore compression brace for 23 hours a day and additionally attended a physiotherapy program for a period of 12 weeks; *independent sample t test, chi square test 


\begin{tabular}{|c|c|c|c|}
\hline Parameters & $\begin{array}{l}\text { Group } 1 \\
\text { [mean } \pm \text { standard deviation or } \mathrm{n} \\
\text { (frequency \%)], } \mathrm{n}: 10\end{array}$ & $\begin{array}{l}\text { Group } 2 \\
\text { [mean } \pm \text { standard deviation or } \mathrm{n} \\
\text { (frequency \%)], } \mathrm{n}: 13\end{array}$ & $\mathbf{p}$ \\
\hline Scoliosis & $6(60 \%)$ & $7(53.84 \%)$ & 0.510 \\
\hline \multicolumn{4}{|c|}{ Brace Side effect } \\
\hline Pain & $7(70 \%)$ & $6(46.15 \%)$ & 0.249 \\
\hline $\begin{array}{l}\text { Shortness of } \\
\text { breath }\end{array}$ & $2(20 \%)$ & $1(7.69 \%)$ & 0.704 \\
\hline Irritation & $5(50 \%)$ & $2(15.38 \%)$ & 0.340 \\
\hline Wound & $3(30 \%)$ & 1 (7.69\%) & 0.412 \\
\hline \multicolumn{4}{|c|}{$\begin{array}{l}\text { PC: Pectus carinatum; Group 1, patients who wore compression brace for } 23 \text { hours a day for a period } \\
\text { of } 12 \text { weeks; Group } 2 \text {, patients who wore compression brace for } 23 \text { hours a day and additionally } \\
\text { attended a physiotherapy program for a period of } 12 \text { weeks; *independent sample t test, chi square } \\
\text { test }\end{array}$} \\
\hline
\end{tabular}


Table 2

Evaluation parameters before and after treatment

\begin{tabular}{|c|c|c|c|c|c|c|c|}
\hline \multirow[t]{2}{*}{ Assessment } & \multirow[t]{2}{*}{ Group } & \multirow{2}{*}{$\begin{array}{l}\text { Baseline } \\
\text { mean }\end{array}$} & \multicolumn{2}{|c|}{12 weeks } & \multirow{2}{*}{$\begin{array}{l}\text { Effect } \\
\text { size }\end{array}$} & \multirow{2}{*}{$\begin{array}{l}P^{\star} \\
\text { within- } \\
\text { group }\end{array}$} & \multirow{2}{*}{$\begin{array}{l}\mathrm{P}^{\star *} \\
\text { Betweer } \\
\text { group }\end{array}$} \\
\hline & & & Mean & $\begin{array}{l}\text { Within-group } \\
\text { score change }\end{array}$ & & & \\
\hline \multirow{3}{*}{$\begin{array}{l}\text { Maximum } \\
\text { protrusion degree }\end{array}$} & & & & & & & \multirow[t]{3}{*}{0.026} \\
\hline & $\begin{array}{l}\text { Group } \\
1\end{array}$ & $\begin{array}{l}26.73 \pm \\
7.07\end{array}$ & $\begin{array}{l}13.10 \\
\pm 10.20\end{array}$ & $-13.63 \pm 5.85$ & 1.93 & $\langle .001$ & \\
\hline & $\begin{array}{l}\text { Group } \\
2\end{array}$ & $\begin{array}{l}23.28 \pm \\
5.54\end{array}$ & $\begin{array}{l}4.58 \pm \\
4.48\end{array}$ & $-19.02 \pm 5.55$ & 3.43 & $<.001$ & \\
\hline
\end{tabular}

Craniocaudal length

0.639

\begin{tabular}{llllll} 
Group & $22.09 \pm$ & 21.18 & $-0.50 \pm 2.01$ & 0.11 & 0.453 \\
1 & 4.43 & \pm 4.10 & & & \\
\hline Group & $26.61 \pm$ & 24.37 & $-0.82 \pm 1.50$ & 0.18 & 0.039 \\
2 & 4.32 & \pm 5.16 & & & \\
\hline
\end{tabular}

Lateral length

\begin{tabular}{llllll} 
Group & $17.15 \pm$ & 16.37 & $-0.78 \pm 0.48$ & 0.4 & $<$ \\
1 & 1.95 & \pm 1.83 & & & $\mathbf{0 . 0 0 1}$ \\
\hline Group & $16.60 \pm$ & 14.90 & $-1.70 \pm 0.80$ & 0.92 & $\mathbf{<}$ \\
2 & 1.83 & \pm 2.39 & & & $\mathbf{0 . 0 0 1}$
\end{tabular}

T1

\begin{tabular}{|c|c|c|c|c|c|}
\hline $\begin{array}{l}\text { Group } \\
1\end{array}$ & $\begin{array}{l}9.82 \pm \\
1.40\end{array}$ & $\begin{array}{l}9.87 \pm \\
1.39\end{array}$ & $-0.05 \pm 0.12$ & 0.03 & 0.167 \\
\hline $\begin{array}{l}\text { Group } \\
2\end{array}$ & $\begin{array}{l}10.32 \pm \\
1.56\end{array}$ & $\begin{array}{l}10.35 \\
\pm 2.30\end{array}$ & $-0.03 \pm 0.12$ & 0.02 & 0.203 \\
\hline
\end{tabular}

T2

\begin{tabular}{llllll} 
Group & $13.82 \pm$ & 13.54 & $-0.03 \pm 0.08$ & 0.02 & 0.167 \\
1 & 1.99 & \pm 2.16 & & & \\
\hline Group & $15.21 \pm$ & 15.29 & $-0.06 \pm 0.23$ & 0.03 & 0.298 \\
2 & 2.35 & \pm 2.33 & & & \\
\hline
\end{tabular}

T3

Group 1, patients who wore compression brace for 23 hours a day for a period of 12 weeks; Group 2, patients who wore compression brace for 23 hours a day and additionally attended a physiotherapy program for a period of 12 weeks; T1, measured length between upper edge of manubrium - spinous process of the same level vertebra; T2, measured length between Angulus ludovici - spinous process of the same level vertebra, T3, measured length between the most prominent point of sternumspinous process of the same level vertebra; Pectus carinatum severity, T3/T1 $\times 100$ (\%); NYPR, New York Posture Rating Scale; mean \pm standard deviation, *paired-sample t test, $\star \star 2 \times 2$ way mixed ANOVA 


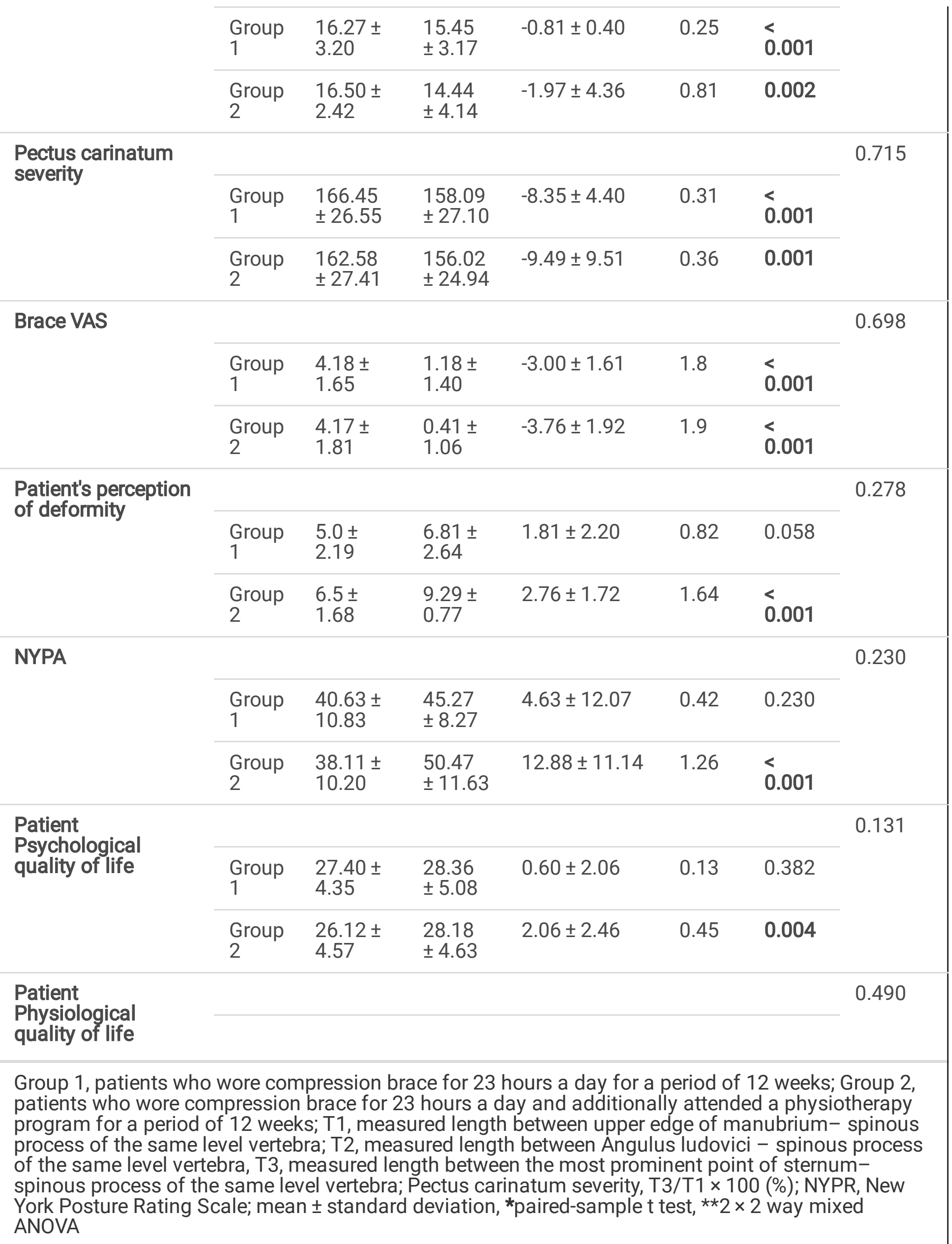




\begin{tabular}{|c|c|c|c|c|c|c|c|}
\hline & $\begin{array}{l}\text { Group } \\
1\end{array}$ & $\begin{array}{l}11.00 \pm \\
0.81\end{array}$ & $\begin{array}{l}11.27 \\
\pm 1.00\end{array}$ & $0.27 \pm 0.79$ & 0.24 & 0.443 & \\
\hline & $\begin{array}{l}\text { Group } \\
2\end{array}$ & $\begin{array}{l}11.68 \pm \\
2.60\end{array}$ & $\begin{array}{l}11.31 \\
\pm 1.89\end{array}$ & $0.37 \pm 2.50$ & 0.14 & 0.557 & \\
\hline \multirow{3}{*}{$\begin{array}{l}\text { Parents } \\
\text { Psychological } \\
\text { quality of life }\end{array}$} & & & & & & & \multirow[t]{3}{*}{0.282} \\
\hline & $\begin{array}{l}\text { Group } \\
1\end{array}$ & $\begin{array}{l}25.90 \pm \\
3.03\end{array}$ & $\begin{array}{l}25.72 \\
\pm 3.63\end{array}$ & $-0.50 \pm 0.85$ & 0.16 & 0.096 & \\
\hline & $\begin{array}{l}\text { Group } \\
2\end{array}$ & $\begin{array}{l}27.20 \pm \\
4.44\end{array}$ & $\begin{array}{l}26.80 \\
\pm 4.53\end{array}$ & $0.40 \pm 2.47$ & 0.09 & 0.541 & \\
\hline \multirow{3}{*}{$\begin{array}{l}\text { Parents } \\
\text { Physiological } \\
\text { quality of life }\end{array}$} & & & & & & & \\
\hline & $\begin{array}{l}\text { Group } \\
1\end{array}$ & $\begin{array}{l}14.80 \pm \\
2.04\end{array}$ & $\begin{array}{l}15.36 \\
\pm 2.46\end{array}$ & $0.60 \pm 1.83$ & 0.29 & 0.329 & \multirow[t]{2}{*}{0.359} \\
\hline & $\begin{array}{l}\text { Group } \\
2\end{array}$ & $\begin{array}{l}14.60 \pm \\
2.66\end{array}$ & $\begin{array}{l}14.40 \\
\pm 1.95\end{array}$ & $0.20 \pm 2.24$ & 0.07 & 0.735 & \\
\hline \multicolumn{8}{|c|}{$\begin{array}{l}\text { Group 1, patients who wore compression brace for } 23 \text { hours a day for a period of } 12 \text { weeks; Group } 2 \text {, } \\
\text { patients who wore compression brace for } 23 \text { hours a day and additionally attended a physiotherapy } \\
\text { program for a period of } 12 \text { weeks; T1, measured length between upper edge of manubrium- spinous } \\
\text { process of the same level vertebra; T2, measured length between Angulus ludovici }- \text { spinous process } \\
\text { of the same level vertebra, T3, measured length between the most prominent point of sternum- } \\
\text { spinous process of the same level vertebra; Pectus carinatum severity, T3/T1 } 100 \text { ( } 10 \text { ); NYPR, New } \\
\text { York Posture Rating Scale; mean } \pm \text { standard deviation, *paired-sample t test, ** } 2 \times 2 \text { way mixed } \\
\text { ANOVA }\end{array}$} \\
\hline
\end{tabular}

VAS scores for pain experienced during use of brace showed significant reduction in both groups $(\mathrm{p}<$ 0.001), whereas only Group 2 had a statistically significant increase in patients' deformity perception and NYPR scores $(p<0.001$, Table 2$)$.

Parent life quality did not show a significant change in either of the groups $(p>0.05)$, whereas patient psychological life quality showed a significant improvement in only Group $2(p=0.004)$. Physiological life quality did not show a statistically significant change in either of the groups $(p>0.05$, Table 2$)$.

Whenever any of the above measurements showed a statistically significant change, Group 2 always had higher $E S$ value $(E S>0.36)$.

In comparison of GRC scores between the groups, 'better' and 'much better' responses were significantly more frequent in Group 2 (Table 3). 
Table 3

Global Rating of Change scores after treatment

\begin{tabular}{|c|c|c|c|}
\hline Global Rating of Change & $\begin{array}{l}\text { Group } 1 \\
\text { [n (frequency \%)], n:10 }\end{array}$ & $\begin{array}{l}\text { Group } 2 \\
\text { [n (frequency \%)], n:13 }\end{array}$ & $\mathrm{P*}$ \\
\hline-2 (Much worse) & 0 & 0 & 0.003 \\
\hline-1 (worse) & 0 & 0 & \\
\hline 0 (same) & $2(20 \%)$ & 0 & \\
\hline 1 (better) & $7(70 \%)$ & $3(23.07 \%)$ & \\
\hline 2 (much better) & $2(20 \%)$ & $10(76.92 \%)$ & \\
\hline
\end{tabular}

\section{Discussion}

In this study, we aimed to investigate the effect of physiotherapy protocol applied in conjunction with brace treatment in patients with PC. Although both study groups showed improvement in PC protrusion compared to baseline, the group that received adjunct physiotherapy had higher effect scores.

Additionally, maximum protrusion degree and lateral length values were statistically better following treatment in the group receiving adjunct physiotherapy when compared to the group treated with a brace only. In addition, patients' deformity perception, posture, patient psychological life quality, and treatment satisfaction scores were significantly better in the physiotherapy group.

$\mathrm{PC}$ is a common pediatric condition characterized by idiopathic overgrowth of the costal cartilages that results in protrusion of sternum. The severity of this abnormality often worsens during the growth spurt in puberty. Overall prevalence of PC is $0.6 \%$, and it is more common among males [29]. We included 30 male patients in the present study. This was both because of the fact that it is more prevalent among males, and also because we were worried that breast development in females would affect the study results. One of the limitations of our study is that women with PC cannot be included in the study.

Consistent with the literature, the most frequent complaint of patients with PC in our study was aesthetic concerns $(100 \%)$, although the disease is known to cause more than simple aesthetic problems. The disease is also responsible for various physical signs and symptoms, as well as important psychological effects. These problems tend to deteriorate during pubertal growth spurts and even in the adult life [4]. In our study, there were two patients in each group complaining of mild dyspnea as physical symptoms in addition to the aesthetic problem. Kravarusic et al. [11] recommended early puberty for initiation of the brace treatment. When we examine the patient ages, we see that most patients sought treatment following the onset of puberty. 
One non-surgical approach involving the use of dynamic chest compression orthotics was first proposed by Haje and Raymundo in 1979, and further developed in 1988 [30]. The brace was designed for maintaining continuous compression in order to stimulate remodeling of bone and cartilage. This places the maximum external force over the most prominent point of sternal protrusion. These early braces resembled a scoliosis jacket support and were not very popular among patients because of the discomfort they caused and the difficulty of adjustment. However, changes introduced to the devices, such as adjustable fasteners that allow patients to control the pressure considerably, improved compliance and efficacy of this intervention [11].

Beginning from 1970's, patients with PC have been treated using compression braces that restore the normal thoracic shape by applying external compression over the thoracic wall. This method yielded predominantly favorable outcomes [10-15, 30-34]. Nevertheless, treatment give-up rates due to noncompliance, in particular, were high and reached $40 \%$ in some studies $[10,14,30]$. Major reasons for giving up use of the brace included embarrassment, sweating, and various adverse effects such as rash and skin ulcers caused by the pressure. In our study, pain and/or adverse effects were observed in the rates of $70 \%$ in Group 1 and $46 \%$ in Group 2. Five patients in Group $1(1 / 3)$ left the study due to embarrassment and adverse effects, whereas 2 patients left the study in the physiotherapy group. Moreover, we observed that training the patients on performing inspiration to lung lobes below the level of brace with respiratory exercises helped to alleviate discomfort and pain. However, we did not document this because most of the patients in both groups did not have the information recorded. This was a limitation of the present study, and we recommend future studies to elaborate on this subject.

Banever et al. [13] used brace in 30 patients, and observed that a third of their patients abandoned treatment. Kravarusic et al. [11] applied the Calgary protocol (23 hours) as in our study, and observed that 19 of the 24 patients continued treatment. Beer et al. [12] reported that 78 of their patients completed brace treatment, while 27 patients abandoned it. Due to the low compliance of patients, they underscored the importance of careful and regular follow-up for the success of compression braces. In our study, we observed that the group that received adjunct physiotherapy showed better compliance since this group was under more careful follow-up. This is also supported by studies reporting that braces should be applied concurrently with individually-tailored exercise programs to increase compliance with the treatment.

Kravarusic et al. [11] observed that compression braces yielded favorable results in the short-term comparable to surgical treatment. In addition, Beer et al. [12] reported that 78 of their patients completed brace treatment and had favorable results; Lee et al. [14] studied 98 patients and documented that brace treatment reduced protrusion degree when patients showed good compliance. In our study, we observed statistically significant improvement in external chest measurements such as maximum protrusion degree, lateral length, T3 value, and PC severity in both groups at the 12th week. However, the effect size of treatment was higher in the group receiving adjunct physiotherapy, and maximum protrusion angle and lateral length measurement showed greater improvement in this group compared to the brace-only group. 
Similar to our study, Banever et al. [13] applied an orthotics and exercise program that included chest wall muscle-strengthening exercises and deep respiration exercises to 30 pediatric patients. They reported that 20 patients continued treatment, and complete correction was achieved in 15 patients within a 16 month period. However, they mostly focused on the effect of brace rather than exercise, since they did not have an orthotics-only group in their study. Marcelo Martinez-Fero et al. [4] stated that the physiotherapist lied in the center of non-surgical treatment, and was an essential member of the healthcare team for improvement of the posture. Giray et al. [15] applied exercise treatment to 3 groups of patients during a period of one month. The groups used brace either for 8 hours, 23 hours, or not at all. Similar to our results, the greatest improvement was observed when patients used the brace for 23 hours. Nonetheless, favorable results were observed in all three groups. This suggests that exercise alone can be effective as well. Although we observed favorable effects in the group that received physiotherapy as an adjunct to brace, we did not have an exercise-only group, which prevented us from making an additional comment on this subject. This is a limitation of the present study.

Recent evidence indicates patients with PC are under risk of disturbed body image and lower life quality. Many patients express emotions of discomfort, shame, embarrassment, anxiety, pain and even depression, which may lead to social isolation. Patients particularly feel chest pain and discomfort while lying prone. Other reported physical signs and symptoms include scoliosis, round shoulders, kyphotic posture, and dyspnea on effort [5]. We also observed postural distortions with high frequency in both groups, and these postural distortions were markedly corrected in the group receiving adjunct physiotherapy. Regarding life quality, the patient psychological life quality scores were observed to show better improvement in the physiotherapy group. No significant change was observed regarding patient physiological life quality or parental life quality scores. We believe this may be because of the short treatment period that prevented us to see the reflections of favorable effects on life quality.

Most clinicians request their patients to evaluate their health improvement in time, and then use this data to guide their treatment decisions. GRC scales provide means to attain these data in rapid, flexible, and efficient way [23]. We used a 5-point GRC scale in our study, and observed that patients in the physiotherapy group responded significantly and more frequently as 'much better' and 'better' compared to the group treated with the brace alone. This can be related to the higher effect size of treatment and greater compliance in the physiotherapy group.

\section{Conclusion}

One limitation of the present study is the relatively short treatment period. Long-term effects of our treatments should be investigated in future studies. Nonetheless, we believe brace treatment should be administered in conjunction with proper cardiopulmonary and musculoskeletal exercise programs based on our results documenting additional improvements and satisfaction in the physiotherapy group.

\section{Abbreviations}


PC: Pectus carinatum; T1: measured length between upper edge of manubrium- spinous process of the same level vertebra; T2: measured length between Angulus ludovici - spinous process of the same level vertebra, T3: measured length between the most prominent point of sternum- spinous process of the same level vertebra; NYPR,: New York Posture Rating Scale

\section{Declarations}

\section{Acknowledgments}

Not applicable.

\section{Authors' contributions}

Concept - N.A., I. A., M.Y.; Design - N.A., I. A., M.Y.;Supervision- N.A., M.Y.; Resource - N.A., I. A., M.Y.; Materials - N.A., I. A., M.Y.;; Data Collection and/or Processing - N.A., I.A.; Analysis and/or InterpretationN.A., I. A., M.Y..; Literature Search - N.A., I. A., M.Y.; Writing- N.A., M.Y.; Critical Reviews - N.A., İ. A., M.Y.

\section{Funding}

None declare

\section{Availability of data and materials}

All data generated or analyzed during this study are included in this published article.

\section{Ethics approval and consent to participate}

The Research Ethics Committee of Acıbadem University and Acıbadem Healthcare Group has approved the study (ATADEK-2019-14/11) and registered as a clinical trial (registration no: NCT04167800).

\section{Consent for publication}

All the authors agree to the publication of this manuscript.

\section{Competing interests}

The authors declare that they have no competing interests

\section{Author details}

${ }^{1}$ Department of Physiotherapy and Rehabilitation, Faculty of Health Sciences, Acibadem Mehmet Ali Aydinlar University, Istanbul, Turkey

${ }^{2}$ Chest Wall Deformities and Pectus Association, Department of Physiotherapy and Rehabilitation, Istanbul, Turkey 
${ }^{3}$ Chest Wall Deformities and Pectus Association, Department of Thoracic Surgery, Istanbul, Turkey

\section{References}

1. Blanco FC, Elliott ST, Sandler AD, et al. Management of congenital chest wall deformities. Semin Plast Surg. 2011; 25:107-16.

2. Frey AS, Garcia VF, Brown RL, et al. Nonoperative management of pectus carinatum. J Pediatr Surg. 2006; 41(1):40-45.

3. Kuhn MA, Nuss D. Pectus deformities. In: Mattei P (ed). Fundamentals of Pediatric Surgery, Springer, New York, 2011: 313-22.

4. Martinez-Ferro M, Munzon GB, Fraire $C$, et al. Non-surgical treatment of pectus carinatum with the FMF® Dynamic Compressor System. J Vis Surg. 2016; 2.

5. Steinmann C, Krille S, Mueller A, et al. Pectus excavatum and pectus carinatum patients suffer from lower quality of life and impaired body image: a control group comparison of psychological characteristics prior to surgical correction. Eur J Cardiothorac Surg. 2011; 40(5);1138-1145.

6. Ravitch MM. The operative treatment of pectus excavatum. Ann Surg. 1949; 129:429-44.

7. Varela $\mathrm{P}$, Torre $\mathrm{M}$. Thoracoscopic cartilage resection with partial perichondrium preservation in unilateral pectus carinatum: preliminary results. J Pediatr Surg. 2011; 46(1):263-266.

8. Abramson H, D'Agostino J, Wuscovi S, et al. A 5-year experience with a minimally invasive technique for pectus carinatum repair. J Pediatr Surg. 2009; 44:118-23.

9. Desmarais TJ, Keller MS. Pectus carinatum. Curr Opin Pediatr. 2013; 25(3):375-381

10. Kang DY, Jung J, Chung S, et al. Factors affecting patient compliance with compressive brace therapy for pectus carinatum. Interact Cardiovasc Thorac Surg. 2014;19(6): 900-903.

11. Kravarusic D, Dicken BJ, Dewar R, et al. The Calgary protocol for bracing of pectus carinatum: a preliminary report. J Pediatr Surg. 2006; 41:923-926.

12. de Beer SA, Gritter M, de Jong JR, et al. The dynamic compression brace for pectus carinatum: intermediate results in 286 patients. Ann Thorac Surg. 2017; 103:1742-1749.

13. Banever GT, Konefal Jr SH, Gettens K, et al. Nonoperative correction of pectus carinatum with orthotic bracing. J Laparoendosc Adv. 2006; 16:164-167.

14. Lee RT, Moorman S, Schneider M, et al. Bracing is an effective therapy for pectus carinatum: interim results. J Pediatr Surg. 2013; 48:184-190.

15. Giray E, Ermerak N, Bahar YO, et al. A Comparative Study on Short-Term Effects of Compression Orthosis and Exercises in the Treatment of Pectus Carinatum: A Randomized Controlled Pilot Feasibility Trial. Eur J Pedıatr Surg. 2020.

16. Borg GA. Psychophysical bases of perceived exertion. Med Sci Sports Exerc. 1982;14(5):377-81.

17. Riebe D. ACSM's guidelines for exercise testing and prescription. Wolters Kluwer, 2018 
18. Akkaş Y, Peri, N. G, Koçer B, Gülbahar G, Aksakal FNB. The prevalence of chest wall deformity in Turkish children. Turk J Med ScI. 2018; 48(6):1200-1206.

19. Ewert F, Syed J, Wagner S, et al. Does an external chest wall measurement correlate with a CT-based measurement in patients with chest wall deformities?. J Pediatr Surg. 2017; 52(10):1583-1590.

20. Canavan PK, Cahalin L. Integrated physical therapy intervention for a person with pectus excavatum and bilateral shoulder pain: a single-case study. Arch Phys Med Rehabil. 2008; 89(11);2195-2204.

21. Price DD, McGrath PA, Rafii A, Buckingham B, et al. The validation of visual analogue scales as ratio scale measures for chronic and experimental pain. Pain. 1983;17:45-56.

22. Magee DJ. Orthopedic Physical Assessment. Gait Assessment. Chapter13. W.B. Saunders Company, Toronto. 1987; 362-376.

23. Kamper SJ, Maher CG, Mackay G. et al. Global rating of change scales: a review of strengths and weaknesses and considerations for design. J Man Manip Ther. 2009; 17(3):163-170.

24. Lawson ML, Cash TF, Akers R, et al. A pilot study of the impact of surgical repair on disease-specific quality of life among patients with pectus excavatum. J Pediatr Surg. 2013; 38(6):916-8.

25. Krasopoulos G, Dusmet M, Ladas G, Goldstraw P. et al. Nuss procedure improves the quality of life in young male adults with pectus excavatum deformity. Eur J Cardıo-Thorac 2006; 29:1-5

26. de Vet HC, Terwee CB, Bouter LM, et al. Current challenges in 490 clinimetrics. J Clin Epidemiol. 2003; 56:1137-41.

27. Norman GR, Stratford P, Regehr G, et al. Methodological problems 491 in the retrospective computation of responsiveness to change: 492 the lesson of Cronbach. J Clin Epidemiol. 1997; 50:869-79.

28. Shamberger RC, Welch KJ. Surgical repair of pectus excavatum. J Pediatr Surg. 1998; 23(7):615-622.

29. Haje SA, Bowen JR. Preliminary results of orthotic treatment of pectus deformities in children and adolescents. J Pediatr Orthop. 1992; 12:795-800.

30. Lee SY, Lee SJ, Jeon CW, Lee CS, Lee KR, et al. Effect of the compressive brace in pectus carinatum. Eur J Cardiothorac Surg. 2008;34:146-9.

31. Egan JC, DuBois JJ, Morphy M, et al. Compressive orthotics in the treatment of asymmetric pectus carinatum: a preliminary report with an objective radiographic marker. J Pediatr Surg. 2000; 35:1183-6.

32. Stephenson JT, Du Bois J. Compressive orthotic bracing in the treatment of pectus carinatum: the use of radiographic markers to predict success. J Pediatr Surg. 2008; 43:1776-80.

33. Jung J, Chung SH, Cho JK, et al. Brace compression for treatment of pectus carinatum. Korean $\mathrm{J}$ Thorac Cardiovasc Surg. 2012; 45:396-400.

34. Colozza S, Butter A. Bracing in pediatric patients with pectus carinatum is effective and improves quality of life. J Pediatr Surg. 2013;48:1055-9. 